Apidologie, 1976, 7 (1), 39-47.

\title{
WIRKUNG VON ZWEI JUVENOIDEN AUF BIENENVÖLKER (APIS MELLIFERA L.)
}

\author{
Action de deux juvénoüdes sur des colonies d'abeilles (Apis mellifera L.) \\ Ivan HRDÝ und Dimitrij ŠKROBAL \\ Entomologisches Institut der Tschechoslowakischen Akademie der Wissenschaften, \\ Viničná 7, 12800 Praha 2, Tschechoslowakei und \\ Institut für Bienenkunde, Station Prosějtov, Vodní ulice 31, 79600 Prostějov, Tschechoslowakei
}

\section{SUMMARY}

EFFECT OF TWO JUVENOIDS ON HONEY BEE COLONIES (Apis mellifera L.)

The effectiveness of juvenile hormone analogues (JHA or juvenoids) on honey bees has been tested : (1) JHA in sugar solution was administered to small colonies of the honey bee, (2) greenhouses (individual treated plot $36 \mathrm{~m}^{2}$ ) with radish plants in blossom were infested by two honey bee colonies. JHA as emulsions were applied by spraying. The effects of juvenoids (I) methyl (2 E) 11-chloro-3,7,11-trimethyl-2-dodecenoate and (II) ethyl (2 E, 4 E)-3,7,11trimethyl-2,4-dodecadienoate were compared with those of the conventional insecticide Phosdrin (mevinphos).

Despite the quantity of juvenoids administered (individual honey bee colonies consumed 7.5-15.5 g of active compound) no harmful effect on the adult workers was observed. However, the brood was affected deleteriously, and after 32 days of JHA administration there was no brood in the sections. Two of the four queens died. The worker bees in the greenhouse were also not harmfully affected. However, some changes in brood rearing were recorded. The decrease in brood quantity in JHA treated colonies was higher in comparison with controls. The egg deposition of queens was not directly affected but it was probably stimulated. Some losses of worker bees were caused by the closed greenhouse environment, but the greatest reduction of worker bees was observed in Phosdrin treated colonies.

\section{ZUSAMMENFASSUNG}

Die Wirkung der Juvenilhormonanaloge (JHA oder Juvenoide) auf die Honigbiene wurde getestet : (1) durch Verfütterung der in Zuckerlösung emulgierten JHA an Bienenvölker, (2) in Glashaustesten mit blühenden und.JHA-behandelten Radieschenpflanzen (Versuchsfläche $36 \mathrm{~m}^{2}$ ) und mit je zwei darauf angesetzten Bienenvölkern, wobei die JHA (I) Methyl (2 E) 11- 
chloro-3,7,11-trimethyl-2-dodecenoate und JHA (II) Ethyl (2 E, 4. E)-3,7,11,-trimethyl-2,4dodecadienoate als Emulsion durch Bespritzung appliziert und Wihre irkung mit dem klassischen Insektizid Phosdrin (mevinphos) verglichen wurden.

Trotz der grossen Menge der den Bienen verfütterten Juvenoide (die einzelnen Bienenvölkchen konsumierten 7,5 bis $15,5 \mathrm{~g}$ des aktiven Stoffes) wurde keine schädliche Wirkung auf die Arbeitsbienen festgestellt. Dagegen wurde die Brut betroffen : nach einem Monat (32 Tagen) der JHA-Verfütterung war in keinem der vier Bienenvölkchen noch Brut vorhanden. Zwei von vier Königinnen wurden tot gefunden.

Beim Glashausversuch wurden die Arbeitsbienen ebenfalls nicht betroffen, jedoch ergab sich ein Einfluss auf die Brutaufzucht. Im Vergleich zur Kontrolle wurde bei den Bienenvölkern von der mit JHA-behandelten Parzelle eine Verminderung der Brutfläche, besonders der offenen Brut, beobachtet. Ein direkter störender Einfluss auf die Eiablage der Königinnen war nicht festzustellen, es scheint eher, dass eine Stimulation hervorgerufen wurde. Infolge der Glashaushaltung der Bienenvölker verminderte sich die Anzahl der Sammelbienen. Der höchste Verlust trat jedoch nach Behandlung mit Phosdrin ein, zweifellos infolge toxischer Wirkung.

\section{EINLEITUNG}

Die Juvenilhormonanaloge (JHA oder Juvenoide) werden als perspektivische Stoffe der Insektenbekämpfung angesehen. Ihre Wirkung auf die Honigbiene wurde durch topikale Applikation einiger JHA bei empfindlichen Stadien von Apis mellifera festgestellt (Ž́ÁreK \& Haragsim, 1974), wobei sich Entwicklungsstörungen bemerkbar machten. Die Bedeutung des Juvenilhormons für die Kastendetermination bei der Honigbiene wurde in der Arbeit von Wintz \& BeEtsna (1972) herausgestellt und eingehender dann von Wirtz (1973) beschrieben. Über die Wirkung der den Bienenvölkern in Nahrung angebotenen Juvenoide machten Haragsim \& Ž́d́reK 1973) und HrDÝ (1973) vorläufige Mitteilungen. Ủber die Einwirkung auf die Hymenopteren im allgemeinen ist nur sehr wenig bekannt, wie auch aus der Gesamtübersicht über die biologische Wirkung von JHA auf verschiedene Insektengruppen hervorgeht (StaAL, 1972). Mit Rücksicht auf die Bedeutung der Honigbiene im Ökosystem sind jedoch eingehendere Kenntnisse auf diesem Gebiet erforderlich.

\section{MATERIAL UND METHODIK}

Für alle Untersuchungen (Verfütterungs- und Glashausversuche) wurde die im Institut für Bienenkunde, Station Prostějov, gezüchtete Honigbiene, Apis mellifera carnica, Stamm Troiseck, verwendet.

Applizierte Stoffe : (I) Methyl (2 E) 11-chloro-3,7,11-trimethyl-2-dodecenoate (JARoLím et al., 1969); (II) hydroprene : Ethyl (2 E, 4 E)-3,7,11-trimethyl-2,4-dodecadienoate * (HENRICK, 1972). Als klassisches Insektizid wurde im Glashausversuch Phosdrin (mevinphos) verwendet. In den Kontrollen beider Versuchsserien wurden Emulgatoren benutzt.

\section{Verfütterungsversuch}

Kleine Bienenvölkchen wurden in Einwabenkästen mit Seitenwänden aus Glas (Rahmenmass $39 \times 24 \mathrm{~cm}$ ) untergebracht und nebeneinander in einen temperaturisolierten Schutzkasten in der Reihenfolge A, B, C, D, E, F eingesetzt, die Fluglöcher abwechselnd zur linken und zur rechten Seite orientiert.

* Zoecon Insect Growth Regulator Altozar TM. 
Die Versuchsbienenvölkchen wurden dem Muttervolk am 1. Juni 1972 entnommen. Die JHA-Applikation begann am nächsten Tag. Die Bienen des Kontrollvölkchens erhielten $50 \%$ ige Zuckerlösung mit $1 \%$ igem Emulgator. Den Versuchsvölkchen wurde $50 \%$ ige Zuckerlösung mit $1 \%$ iger oder $0,5 \%$ iger JHA-Emulsion geboten. Diese Nahrung erhielten sie kontinuierlich im Futterglas.

\section{Glashausversuch}

Wir verfolgten die Wirkung von Juvenoiden, mit denen die Arbeiterinnen beim Sammeln von Nektar und Pollen aus den behandelten Blüten kontaminiert wurden.

Um ein mögliches Abwaschen des applizierten Stoffes bei Regen zu vermeiden, wurden diese Versuche in Glashäusern durchgeführt, in denen Bienenvölker als Bestäuber von zur Samenproduktion bestimmten Radieschenpflanzen dienten. Zwei dieser Glashäuser wurden mit Nylongaze in 4 getrennte Räume mit der Grundfl̈̈che von je 3,25 $\times 11$ m unterteilt. Für Lüftung war durch Gazefenster gesorgt.

In jede der derart vorbereiteten Abteilung kamen am 5. und 7. Juni 1972 zwei Bienenvölker mit legenden Königinnen. Nähere Angaben über die einzelnen Bienenvölker sind aus der Tabelle 2 zu ersehen. Die Bienenvölker erhielten ungefähr $1 \mathrm{~kg}$ Zuckerteig pro Monat. Dieser Nahrungsvorrat war mehr als hinreichend, da während des Versuches ein Teil des Zuckerteigs nicht verbraucht wurde. Die Tracht war zu Beginn des Experiments entsprechend : In der Zeit der Bespritzungen standen die $150 \mathrm{~cm}$ hohen Radieschenpflanzen in voller Blüte und wurden von Bienen gut besucht.

Die einzelnen Versuchsflächen wurden mit je 21 Emulsion bespritzt : JHA in 0,5\% iger Konzentration des Wirkstoffes und Phosdrin in $0,1 \%$ iger Konzentration des Emulsionskonzentrats, und zwar mit einem Vibrationshandspritzgerät (Mistral, Garant) in den Nachmittagsstunden (17 bis 19 Uhr) des 13. Juni 1972.

Die im Glashaus während des Versuches gemessene Temperatur schwankte zwischen $7^{\circ}$ und $35{ }^{\circ} \mathrm{C}$, die relative Feuchtigkeit variierte zwischen 30 bis $100 \%$.

\section{ERGEBNISSE}

\section{Verfütterungsversuch}

Die von den einzelnen Versuchsvölkchen während der 32 Untersuchungstage verfütterte Gesamtmenge der Zuckerlösung ist in der Tabelle 1 angeführt. Die Kontrollvölkchen konsumierten grössere Mengen als die Versuchsvölkchen.

$\mathrm{Zu}$ Beginn und in den ersten Tagen des Versuches enthielten alle Bienenvölkchen Eier, Brut im Larvenstadium und gedeckelte Brut. Während des Experiments wurde in einem Kontrollvölkchen normale Entwicklung ermittelt. Dieses Völkchen (B) befand sich auch nach dem Abschluss des Versuches in sehr gutem Zustand. Das zweite Kontrollvölkchen stellte jedoch die Brutproduktion ein.

In den unter JHA-Einwirkung stehenden Bienenvölkchen wurde festgestellt, dass am 7. Tag des Experiments abgestorbene schwarze Brut von den Arbeiterinnen herausgetragen wurde. Gesunde Larvenstadien fanden sich nicht, wohl aber gedeckelte Brut, und in einem Falle auch Eier. Zwei von vier Königinnen wurden am Flugloch tot gefunden. Am Ende des Experiments verblieben in den JHA-behandelten Bienenvölkchen nur erwachsene Arbeiterinnen oder Königinnen, jedoch keine Brut. 
TAB. 1. - Verfütterungsversuch : Berichı über die Revision der Bienenvälker.

TавL. 1. Expériences de nourrissement : Composition des colonies.

\begin{tabular}{|c|c|c|c|c|c|}
\hline \multirow[b]{2}{*}{$\begin{array}{c}\text { Bienenvölkchen } \\
\text { Teststoff }\end{array}$} & \multirow[b]{2}{*}{$\begin{array}{c}1 \text { Tag vor dem } \\
\text { Versuch }\end{array}$} & \multirow{2}{*}{\multicolumn{2}{|c|}{$\begin{array}{c}\text { Verlauf } \\
\text { des Versuchs }\end{array}$}} & \multicolumn{2}{|c|}{$\begin{array}{c}\text { Verfütterte } \\
\text { Gesamtmenge }\end{array}$} \\
\hline & & & & $\begin{array}{l}\text { Zucker- } \\
\text { lösung } \\
\text { (in ml) }\end{array}$ & $\begin{array}{l}\text { JHA } \\
\text { (in g) }\end{array}$ \\
\hline \multirow{2}{*}{$\begin{array}{l}\text { Colonies } \\
\text { Substance testée }\end{array}$} & \multirow[b]{2}{*}{$\begin{array}{l}1 \text { jour avant } \\
\text { l'expérience }\end{array}$} & \multicolumn{2}{|c|}{$\begin{array}{l}\text { Au cours } \\
\text { de l'expérience }\end{array}$} & \multicolumn{2}{|c|}{$\begin{array}{c}\text { Quantités totales } \\
\text { administrées }\end{array}$} \\
\hline & & $7^{\mathrm{e}}$ jour & $32+$ jour & $\begin{array}{l}\text { Sirop } \\
\text { de sucre } \\
\text { (en } \mathrm{ml} .)\end{array}$ & $\begin{array}{l}\text { JHA } \\
\text { (en g) }\end{array}$ \\
\hline $\begin{array}{l}\text { A } \\
\text { Emulgator (II) }\end{array}$ & $\begin{array}{l}\text { 9, Arbeiterinnen } \\
\text { Brut : Eier } \\
1 \text { tägige, } 5-6 \text { tägige } \\
\text { Larven, gedeckelte } \\
\text { Stadien }\end{array}$ & $\begin{array}{l}? \text {, Arbeiterinnen } \\
\text { Brut : } \\
\text { gedeckelte Stadien }\end{array}$ & $\begin{array}{l}\text { 9, Arbeiterimnen } \\
\text { Brut : } 0\end{array}$ & 2160 & \\
\hline $\begin{array}{l}\text { A } \\
\text { Emulsif }(\mathrm{J} l)\end{array}$ & $\begin{array}{l}\text { क, } \varnothing \\
\text { couvain : œuf } \\
\text { larves d'l jour et de } \\
\mathbf{5 - 6} \text { jours, } \\
\text { stades operculés }\end{array}$ & $\begin{array}{l}\text {, } \\
\text { couvain : } \\
\text { stades operculés }\end{array}$ & $\begin{array}{l}-6 \\
\text { couvain : } 0\end{array}$ & 2160 & \\
\hline $\begin{array}{l}\text { B } \\
\text { Emulgator (I) }\end{array}$ & $\begin{array}{l}\text { ९, Arbeiterinnen } \\
\text { Brut : Eier }\end{array}$ & $\begin{array}{l}\text { 9, Arbeiterinnen } \\
\text { Brut : Larven ver- } \\
\text { schiedenes Alters } \\
\text { gedeckelte Stadien }\end{array}$ & $\begin{array}{l}\text { ९., Arbeiterinnen } \\
\text { Brut : Larven ver- } \\
\text { schiedenes Alters }\end{array}$ & 2190 & \\
\hline $\begin{array}{l}\text { B } \\
\text { Emulsif (I) }\end{array}$ & couvain : oufis & $\begin{array}{l}\text { couvain : larves d'âge } \\
\text { divers, stades operculés }\end{array}$ & $\begin{array}{l}\text {, } \\
\text { couvain : larves d'âge } \\
\text { divers }\end{array}$ & 2100 & \\
\hline$\stackrel{\mathrm{C}}{\mathrm{JHA}}$ (II) $1 \%$ & $\begin{array}{l}\text { ค, Arbeiterinnen } \\
\text { Brut : Eier } \\
\text { 1-5 tägige Larven } \\
\text { gedeckelte Stadien }\end{array}$ & $\begin{array}{l}\text { Q. Arbeiterinnen } \\
\text { Brut : Eier } \\
\text { gedeckelte Stadien }\end{array}$ & 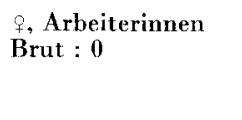 & 1090 & $(10,9)$ \\
\hline $\begin{array}{l}\mathrm{C} \\
\mathrm{JHA} \text { (II) I } \%\end{array}$ & $\begin{array}{l}\text {, } 7 \\
\text { couvain : oufs, larves } \\
\text { d'1 à } 5 \text { jours, stades } \\
\text { operculés }\end{array}$ & $\begin{array}{l}\text { couvain : wufs, } \\
\text { stades operculés }\end{array}$ & $\begin{array}{l}2, \\
\text { couvain : } 0\end{array}$ & 1090 & $(10,9)$ \\
\hline $\begin{array}{l}\mathrm{D} \\
\text { JHA (II) } 0,5 \%\end{array}$ & $\begin{array}{l}9, \text { Arbeiterinnen } \\
\text { Brut : Eier } \\
\text { 1-6 tägige Larven }\end{array}$ & 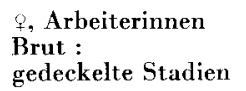 & $\begin{array}{l}\text { tot } \\
\text { Arbeiterinnen } \\
\text { Brut : } 0\end{array}$ & 1490 & $(7,5)$ \\
\hline $\begin{array}{l}\text { D } \\
\text { JHA (1I) } 0,5 \%\end{array}$ & $\begin{array}{l}\text { couvain : coufs } \\
\text { larves d'1 à } 6 \text { jours }\end{array}$ & $\begin{array}{l}+,+ \\
\text { couvain : } \\
\text { stades operculés }\end{array}$ & $\begin{array}{l}\text { r morte, } \\
\text { couvain : } 0\end{array}$ & 1490 & $(7,5)$ \\
\hline $\begin{array}{l}\mathrm{E} \\
\text { JHA (I) } 1 \%\end{array}$ & $\begin{array}{l}\text { 9, Arbeiterinnen } \\
\text { Brut : Eier } \\
1 \text { tägige Larven } \\
\text { gedeckelte Stadien }\end{array}$ & $\begin{array}{l}\text { otot } \\
\text { Arbeiterinnen } \\
\text { Brut : Eier }\end{array}$ & $\begin{array}{l}\text { Arbeiterinnen } \\
\text { Brut : } 0\end{array}$ & 1550 & $(15,5)$ \\
\hline $\begin{array}{l}\mathrm{E} \\
\mathrm{JHA}(\mathrm{I}) 1 \%\end{array}$ & $\begin{array}{l}\text { P, } \\
\text { couvain : ceufs, larves } \\
\text { d'1 jour } \\
\text { stades operculés }\end{array}$ & $\begin{array}{l}9 \text { morte, } 8 \\
\text { couvain : oufs }\end{array}$ & $\begin{array}{l}+ \\
\text { couvain : } 0\end{array}$ & 1550 & $(15,5)$ \\
\hline $\begin{array}{l}\text { F } \\
\text { JHA (I) } 0,5 \%\end{array}$ & $\begin{array}{l}\text { o, Arbeiterinnen } \\
\text { Brut : } 1-6 \text { tägige } \\
\text { Larven, gedeckelte } \\
\text { Stadien }\end{array}$ & $\begin{array}{l}\text { क, Arbeiterinnen } \\
\text { Brut : Eier } \\
\text { gedeckelte Stadien }\end{array}$ & $\begin{array}{l}\text { क, Arbeiterinnen } \\
\text { Brut : } 0\end{array}$ & 1970 & $(9,8)$ \\
\hline JHA (I) $0,5 \%$ & $\begin{array}{l}q, \Varangle \\
\text { couvain : larves de } 1 \text { à } \\
6 \text { jours } \\
\text { stades operculés }\end{array}$ & $\begin{array}{l}\text { couvain : oufs, } \\
\text { stades operculés }\end{array}$ & $\begin{array}{l}\text { ?, } \\
\text { couvain : } \mathbf{0}\end{array}$ & 1970 & $(9,8)$ \\
\hline
\end{tabular}




\section{Glashausversuch}

Die Bespritzungen erfolgten in der Zeit nach Trachtende. Mit Ausnahme von JHA (I) übten die angewandten Stoffe keinen sichtbaren Einfluss auf das Verhalten der Bienen aus. Nach der Behandlung mit dem Juvenoid (I) flogen die Bienen erneut auf Tracht aus und besuchten trotz der späten Nachmittagsstunden noch die Blüten. In den folgenden Tagen konnte jedoch kein Unterschied im Verhalten der Bienen auf den beiden JHA-behandelten Flächen und der Bienen auf den Kontrollflächen festgestellt werden.

Es zeigte sich, dass durch JHA nur die Brut in ihrer Entwicklung, nicht aber die erwachsenen Bienen direkt (toxisch) beeinflusst wurden (siehe Tab. 2). Zehn Tage nach der JHA-Applikation befand sich (mit Ausnahme des Bienenvolkes 017) keine Brut im offenen Larvenstadium. Die durch Juvenoide wahrscheinlich beeinflussten Larven wurden von Arbeiterinnen beseitigt. Nach 16 Tagen war auch keine gedeckelte Brut mehr vorhanden. Die Eiablage der Königinnen blieb jedoch unbeeinflusst, es scheint sogar, dass die Arbeiterinnen infolge des Brutverlustes die Königinnen zu erhöhter Eiablage veranlassten.

In einem Bienenvolk von der mit JHA (II)-behandelten Fläche überlebte ein Teil der Brut, die sich zum gedeckelten Stadium entwickelte. In den der JHA-Wirkung ausgesetzten Bienenvölkern wurden weder erwachsene Bienen noch Larven mit Entwicklungsstörungen gefunden.

Während des Versuches wurde eine allgemeine Verminderung der Arbeiterinnenanzahl beobachtet; gewisse Verluste an Arbeiterinnen sind auf den geschlossenen Raum des Glashauses zurückzuführen. Eine bedeutendere Reduktion der Arbeiterinnenanzahl trat in Bienenvölkern bei den mit Phosdrin behandelten Flächen ein (23. Juni), was von einer direkten toxischen Wirkung von Phosdrin auf die Honighiene zeugt. Auch die Eiablage der Königinnen bei Phosdrin-behandelten Flächen, wie auch bei Kontrollflächen, war markant niedriger als die der Bienenvölker bei JHA-behandelten Flächen (wiederum mit Ausnahme des Bienenvolkes 017, wo eine besonders niedrige Eierzahl beobachtet wurde).

\section{DISKUSSION}

Hohe, in der Nahrung angebotene Gaben von JHA (I) und (II) übten keinen schädlichen Einfluss auf erwachsene Honigbienen aus. Auch die Eiablage der Königinnnen wurde durch die Juvenoide nicht direkt negativ beeinflusst. Der Mangel an Jungbienen infolge des JHA-Effekts konnte sich jedoch später in einer verringerten Eiablage-Aktivität auswirken. 
TAB. 2. - Glashausversuch: Bericht ïber die Revision der Bienenvölker.

TABL. 2. - Expériences sous serre: Composition des colonies.

\begin{tabular}{|c|c|c|c|c|}
\hline $\begin{array}{l}\text { Bienenvolk No. } \\
\text { Colonie no } \\
\text { Teststoff } \\
\text { Substance testée } \\
\text { Königinnenalter } \\
\text { Age de la reine }\end{array}$ & & 13. Juni & 23. Juni & 29. Juni \\
\hline $\begin{array}{l}020 \\
\text { JHA (I) } \\
\text { † } 1970\end{array}$ & 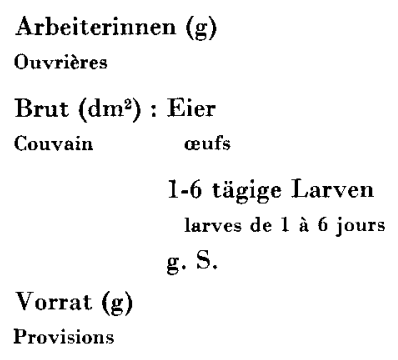 & $\begin{array}{r}500 \\
\\
5 \\
\\
3 \\
\\
12 \\
750\end{array}$ & $\begin{array}{l}400 \\
1,5 \\
0 \\
5 \\
600\end{array}$ & $\begin{array}{r}300 \\
4 \\
0 \\
0 \\
0 \\
250\end{array}$ \\
\hline $\begin{array}{l}04 \\
\text { JHA (I) } \\
\text { 우 } 1969\end{array}$ & 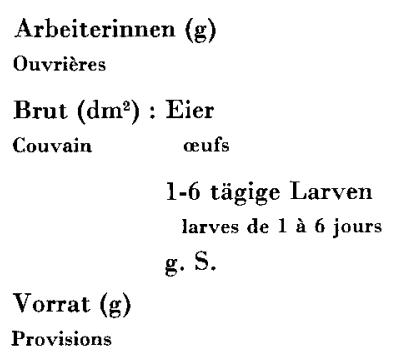 & $\begin{array}{l}300 \\
9,5 \\
7,5 \\
3 \\
250\end{array}$ & $\begin{array}{c}320 \\
2 \\
0 \\
0,75 \\
100\end{array}$ & $\begin{array}{c}250 \\
2 \\
0 \\
0,1 \\
250\end{array}$ \\
\hline $\begin{array}{l}08 \\
\text { JHA (II) } \\
\text { ㅇ } 1970\end{array}$ & 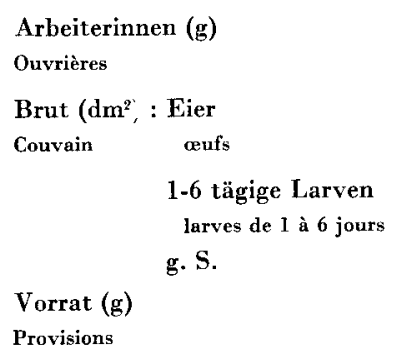 & $\begin{array}{c}500 \\
6,5 \\
6 \\
7 \\
500\end{array}$ & $\begin{array}{c}550 \\
1,5 \\
0 \\
0,1 \\
850\end{array}$ & $\begin{array}{r}350 \\
4 \\
0 \\
0 \\
1000\end{array}$ \\
\hline $\begin{array}{l}017 \\
\text { JHA (II) } \\
q 1971\end{array}$ & 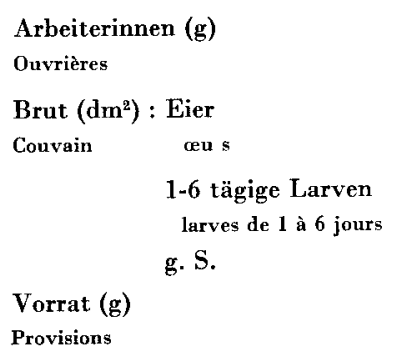 & $\begin{array}{r}0 \\
7 \\
6 \\
600\end{array}$ & $\begin{array}{l}190 \\
0,5 \\
1,5 \\
2,25 \\
200\end{array}$ & $\begin{array}{c}200 \\
0 \\
3 \\
3,5 \\
400\end{array}$ \\
\hline
\end{tabular}




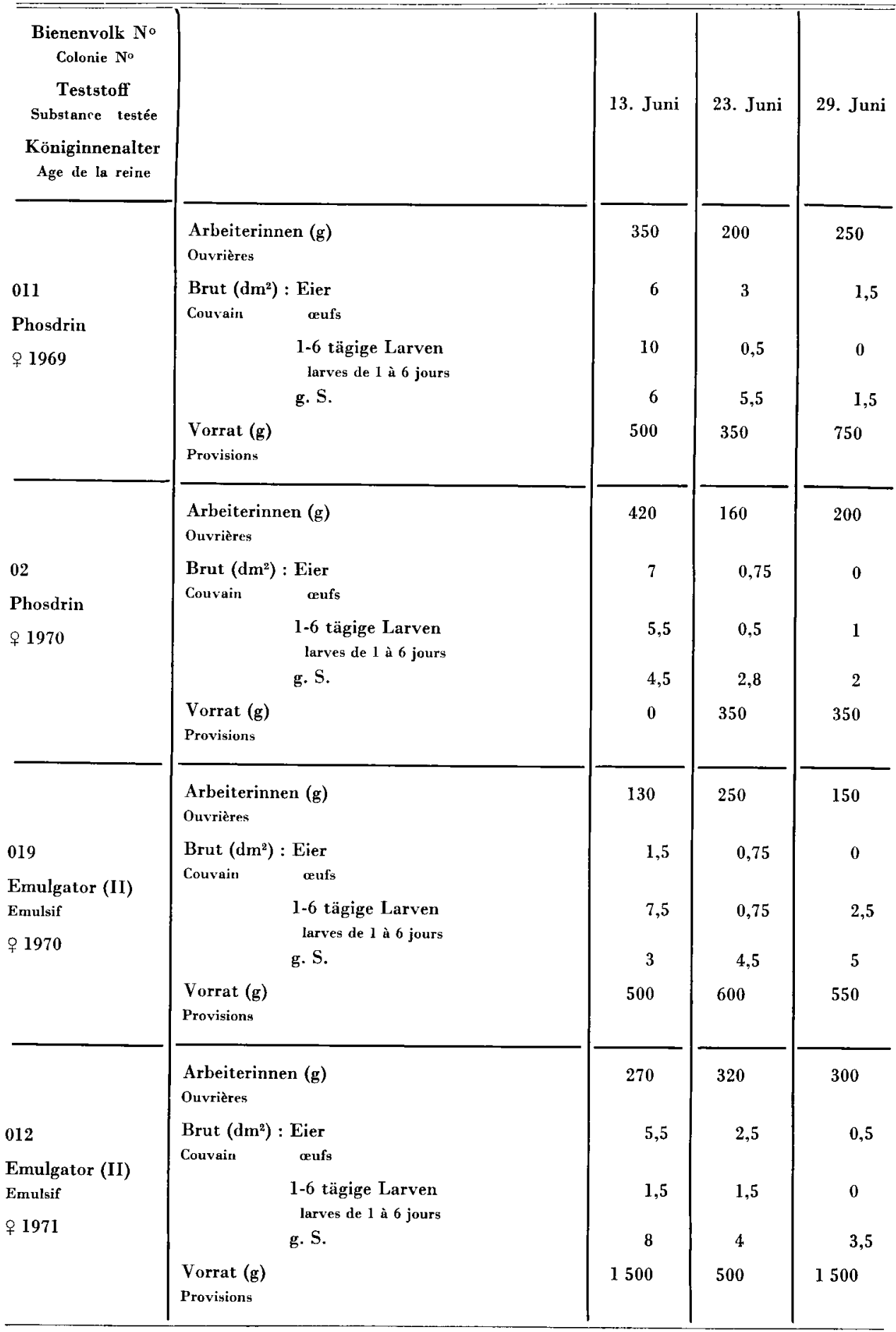

g. S. = gedeckelte Stadien stades operculés. 
Beim Verfütterungsversuch machte sich kein JHA-Sublimationseffekt bemerkbar. Dies folgern wir daraus, dass in den Kontrollvölkchen, die unmittelbar neben den JHA-behandelten Völkchen plaziert waren, keine Veränderungen eintraten, die auf die Einwirkung der Juvenoide zurückgeführt werden könnten.

Auch im Glashausversuch wurde bei den hohen JHA-Konzentrationen ausgesetzten Bienen, die hier im isolierten Raum zur Ernährung an JHAbehandelten Blüten gezwungen waren, keine direkte toxische Wirkung auf die Imagines festgestellt. Durch die JHA-Wirkung kann wahrscheinlich nur die Brut in einem empfindlichen Stadium betroffen werden, wobei dies jedoch schwierig erkennbar ist, da geschädigte Brut von den Arbeiterinnen offensichtlich instinktiv beseitigt wird. Es ist anzunehmen, dass die Bienenvölker nach Erlöschen von JHA-Residuen in ihrer Umwelt und im Bienenstock zur normalen Entwicklung zurückkehren. Drei von den vier der JHA-Wirkung im Glashausversuch ausgesetzten Bienenvölkern zeigten gleiche Reaktion. Ein Bienenvolk fuhr jedoch trotz der JHA-Exposition fort, normale Brut zu entwickeln. Diese Erscheinung kann zur Zeit nicht geklärt werden.

Eingegangen im August 1973*.

Reçu pour publication en août 1973*.

\section{DANK}

Wir danken Frau E. Velccová und Herrn Ing. L. Ševčik für technische Assistenz. Für die Überlassung des Stoffes (I) sind wir Herrn Dr. V. JARoLIM aus dem Institut für Organische Chemie und Biochemie der Tschechoslowakischen Akademie der Wissenschaften, Praha, und des Stoffes (II) der Zoecon Corporation, Palo Alto, zu Dank verpflichtet. Desgleichen auch Herrn Prof. G. Röller, Texas A \& M University und den Herren Dr. G. B. Straal und G. F. LudviK, Zoecon Corporation, für die kritische Durchsicht des Manuskripts.

\section{RÉSUMÉ}

L'action des analogues d'hormone juvénile (JHA ou juvénoïde) a été testée : 1) en administrant aux colonies le JHA en émulsion dans le sirop de sucre, 2) par des expériences sous serre avec 2 colonies d'abeilles et des cultures de radis en fleurs traitées au JHA (surface d'expérimentation : $\left.36 \mathrm{~m}^{2}\right)$. On y a appliqué par arrosage sous forme d'émulsion le JHA (I) dodecenoate de méthyle (2 E) 11-chloro-3, 7, 11-triméthyle-2 et le JHA (II) dodecadenoate d'éthyle (2 E, 4. E)-3, 7, 11-triméthyle-2, 4 et l'on a comparé leur action avec celle de l'insecticide classique Phosdrine (mévinphos).

En dépit de la grande quantité de juvénoïde administrée aux abeilles (chaque ruche a consommé de 7,5 à $15,5 \mathrm{~g}$. de substance active), on n'a pu établir aucune action néfaste sur les ouvrières. Le couvain fut par contre touché; au bout d'un mois (32 jours) de nourrissement il

* Die Veröffentlichung der Arbeit wurde durch den Verlust des Originalmanuskriptes anlässlich der Rücksendung an die Autoren stark verzögert. 
n'y avait plus de couvain dans aucune des 4 colonies. Deux des quatre reines furent trouvées mortes.

Lors des expériences sous serre les ouvrières non plus n'ont pas été touchées, mais une influence sur l'élevage du couvain s'est pourtant fait sentir. On a observé une réduction de la surface de couvain, particulièrement du couvain non operculé, chez les colonies des parcelles traitées au JHA par rapport aux colonies témoins. On n'a pas pu mettre en évidence d'action directement néfaste pour la ponte des reines; il semblerait plutôt qu'une stimulation ait été suscitée. Le nombre de butineuses diminua à la suite du maintien des colonies en serre, mais la plus forte perte intervint après le traitement au Phosdrine, à cause assurément de son action toxique.

\section{LITERATUR}

Haragsim O., ŽDáRek J, 1973. Wirksamkeit einiger als Insektizide angewandter Analoge der Juvenilhormone auf Bienen. Apidologie 4, 188-189.

Henrick C. A., 1972. Belgian Patent No 778-241.

HrdÝ I., 1973. Effect of juvenoids on termites and honeybees. Proc. VII. Congr. I.U.S.S.I., London, 158-161.

Jarolím V., Hejno K., Sehnal F., Šorm P., 1966. Natural and synthetic materials with insect hormone activity. 8 . Juvenile activity of the hormone-type compounds on Galleria mellonella. Life Sci. 8, part II, 831-841.

StaAl S. B., 1972. Biological activity and bioassay of juvenile hormone analogs. Insect Juvenile Hormones, Chemistry and Action, Academic Press, New York-London, 69-94.

WIRTz P., 1973. Differentiation in the honeybee larva. A histological, electron-microscopical and physiological study of caste induction in Apis mellifera mellifera L. Meded. Landbouwhogesch. Wageningen, 73-5, 155 pp.

Wirtz P., Beetsma J., 1972. Induction of caste differentiation in the honeybee (Apis mellifera) by juvenile hormone. Ent. exp. \& appl. 15, 517-520.

Žňárek J., Haragsim O., 1974. Action of juvenoids on metamorphosis of the honey-bee, Apis mellifera. J. Insect Physiol. 20, 209-221. 